\title{
Percepción sociocultural de los pobladores sobre el programa social Juntos en el valle del Mantaro
}

\section{Sociocultural perception of the populators about the social program Juntos in the Mantaro valley}

\author{
${ }^{a}$ Chanca Flores, A.; Bernedo Huachos, E. \\ Facultad de Antropología / Universidad Nacional del Centro del Perú \\ Email: achanca@uncp.edu.pe
}

\section{Resumen}

El artículo tiene por objetivo, conocer la percepción sociocultural de los pobladores no beneficiarios sobre el Programa Nacional de Apoyo Directo a los más Pobres - Juntos en el valle del Mantaro. Es de tipo aplicado y nivel descriptivo, las unidades de análisis consideradas fueron los pobladores no beneficiarios, tanto del género masculino y femenino de los dos distritos seleccionados de cada una de las cuatro provincias del valle del Mantaro. En Concepción: Orcotuna y Chambará; en Chupaca: Ahuac y Chongos Bajo; en Huancayo: Sicaya y Sapallanga; y, en Jauja: Acolla y Sincos. Lugares, donde se aplicó una encuesta de la escala de Likert, a un total de 382 personas mayores de 15 años. El muestreo fue probabilístico y de corte transversal.

Para el procesamiento y análisis de datos, se utilizó la estadística descriptiva, obteniendo como resultado que las percepciones socioculturales de los pobladores no beneficiarios sobre el programa social Juntos son positivas, porque la mayoría de los encuestados perciben aspectos positivos en la ejecución del programa Juntos, el cual viene repercutiendo en el cambio de actitudes y costumbres de los beneficiarios, referidos a la nutrición, higiene, alimentación, educación, salud y organización.

Palabras clave: percepción sociocultural, capital humano, población beneficiaria, inclusión social, programa social

\begin{abstract}
The article aims to know the sociocultural perception of non-beneficiary settlers about the National Program of Direct Support to the Poorest - Juntos in the Mantaro valley. It is of applied type and descriptive level, the units of analysis considered were the non-beneficiary settlers of both the male and female gender of the two selected districts of each of the four provinces of the Mantaro valley. In Concepción: Orcotuna and Chambará; in Chupaca: Ahuac and Chongos Bajo; in Huancayo: Sicaya and Sapallanga; and in Jauja: Acolla and Sincos. Where a survey of the scale of likert was applied to a total of 382 people over 15 years of age. The sampling was probabilistic and cross-sectional.

For the processing and analysis of data, descriptive statistics were used, obtaining as a result that the sociocultural perceptions of the non-beneficiary settlers on the social program Juntos are positive, because most of the respondents perceive positive aspects in the execution of the program together. It has repercussions in the change of attitudes and customs of the beneficiaries, referred to nutrition, hygiene, food, education, health and organization.
\end{abstract}

Keywords: sociocultural perception, human capital, beneficiary population, social inclusion, social program 


\section{Introducción}

Los programas sociales, son iniciativas destinadas a mejorar las condiciones de vida de los pobladores de un país; la mayoría de ellos, son desarrollados por el Estado, que tiene la responsabilidad de atender las necesidades insatisfechas de sus habitantes. De este modo, un gobierno puede poner en marcha planes, programas o proyectos que busquen garantizar el acceso a la educación, campañas de prevención para cuidar la salud o iniciativas para combatir la desnutrición infantil, entre otros. Por ello, en todo el mundo, se observa muchos y variados programas sociales creados según las necesidades y contextos situacionales.

Por otra parte, el programa social Juntos es promovido y ejecutado por el Ministerio de Desarrollo e Inclusión Social (MIDIS), cuyo trabajo fundamental es promover el acceso a los servicios de salud y educación de las familias más pobres del país, con el objetivo de generar capital humano. Fue creado por el Decreto Supremo $N^{o} 062$ en el año 2005, por la Presidencia del Consejo de Ministros, mediante el cual se modifica el Decreto Supremo No 032-2005-PCM, con el que se creó el Programa Nacional de Apoyo Directo a los más Pobres al que denominaron Juntos.

La investigación fue ejecutada durante el año 2019, tuvo como objetivo principal, conocer la percepción sociocultural de los pobladores sobre el programa social Juntos en el valle del Mantaro. Para el cual, las unidades de análisis consideradas fueron los pobladores no beneficiarios tanto del género masculino y femenino de los dos distritos seleccionados de cada una de las cuatro provincias del valle del Mantaro. En Concepción: Orcotuna y Chambará; en Chupaca: Ahuac y Chongos Bajo; en Huancayo: Sicaya y Sapallanga; y, en Jauja: Acolla y Sincos.

El presente informe, fue hecho teniendo en cuenta el cronograma de actividades del proyecto de investigación, por lo que, durante su ejecución se ha dedicado; primero, a la revisión bibliográfica $\mathrm{y} / \mathrm{o}$ archivística, a través de los medios electrónicos y físicos que ha permitido enriquecer el marco teórico; además, de identificar, conocer y analizar las percepciones socioculturales que tienen los pobladores no beneficiarios, respecto al programa social Juntos; a partir de ello, se ha elaborado un instrumento para el recojo de información de campo (encuesta de la escala de Likert), que fue aplicado a un total de 382 pobladores. La encuesta de la escala de Likert, se utilizó porque es uno de los instrumentos de investigación que permite medir y evaluar opiniones, pareceres y actitudes de las personas.

En un contexto, como el nuestro, donde los recursos son escasos, las entidades que financian y ejecutan los programas sociales requieren cada vez más precisión en los resultados de la evaluación. En este sentido, la eva- luación de impacto juega un papel fundamental, porque mide los cambios en las condiciones de vida de los beneficiarios y explica en qué medida estos cambios pueden ser atribuidos al programa.

Año tras año, el Estado invierte grandes sumas de dinero para el financiamiento de los programas sociales, pero no se llega a determinar el impacto en la calidad de vida de las personas a las que van dirigidas. Porque es una intervención unidireccional que se realiza, sin tomar en cuenta la opinión local, por ello, la investigación se torna de importancia para saber la percepción desde la otra mirada.

La investigación permitirá conocer la percepción sociocultural que tienen los pobladores no beneficiarios de los programas sociales implementados en el valle del Mantaro. Resultado que contribuirá para una adecuada toma de desiciones de las principales autoridades y/o funcionarios encargados de las entidades públicas y privadas que operan en esta zona.

\section{Sobre la percepción sociocultural}

En las últimas décadas, el estudio de la percepción viene siendo objeto de creciente interés dentro del campo de la antropología; sin embargo, este interés ha dado lugar a problemas conceptuales, pues el término percepción ha llegado a ser empleado indiscriminadamente para designar a otros aspectos que también tienen que ver con el ámbito de la visión del mundo de los grupos sociales, como es la cosmovisión tanto a nivel individual y/o colectiva (Chanca, 2016).

De allí, la percepción corresponde al plano de las actitudes, los valores sociales o las creencias que han sido creadas y recreadas por los seres humanos, como una forma de apropiación subjetiva de la realidad.

El fenomenólogo francés Merleau - Ponty (1978) consideraba que la conciencia es perceptiva y que esta involucra al cuerpo y el lenguaje.

Bertoni y López (2010) refieren que "Las percepciones sociales del ambiente constituyen sistemas cognitivos en los que es posible reconocer la presencia de opiniones, creencias, valores y normas sobre el ambiente natural de las personas y que van a determinar la orientación actitudinal positiva o negativa para la conservación de la naturaleza”.

Entonces, la percepción social tiene que ser entendida como el proceso mediante el cual las personas interpretan la realidad sociocultural. Así como también, es la forma de ver a los otros, ya sea de forma individual o colectiva y de qué manera interpretamos su actuación.

Por lo señalado líneas arriba, la percepción, para fines del presente estudio, estará enfocado en acopiar la información vertida por los principales actores locales, como parte del sentir, pensar y actuar de los pobladores 
asentados en el valle del Mantaro, sobre el programa social Juntos.

Por otra parte, en nuestro país, como señala Estrada y Perea (2008), la pobreza constituye un grave problema que afecta a un amplio sector de la población y que tiene consecuencias económicas, políticas y sociales. La pobreza impacta en la vida de las personas, limitando sus capacidades naturales, sus oportunidades de desarrollo y el goce de derechos básicos que le son inherentes a la condición humana. Conduce a la desintegración social $y$, en consecuencia, pone en riesgo la propia estabilidad de un país.

Frente a ello, la Contraloría General de la República (2014), refiere que la política social del Estado peruano tiene como propósito principal, la lucha contra la pobreza; para ello, el Estado ha diseñado un conjunto de programas sociales que deben organizarse en función de los ejes orientadores del Plan Nacional para la Superación de la Pobreza, aprobado por Decreto Supremo $\mathrm{N}^{\circ}$ 064-2004-PCM:

En el contexto de superación de la pobreza y en cumplimiento de los Objetivos del Desarrollo Sostenible, los mecanismos de protección social son examinados como herramientas para afrontar la vulnerabilidad y exclusión social. Dentro del amplio campo de protección y la exclusión social, las transferencias de efectivo son efectos que atraen mucha atención e interés y han sido desarrolladas en América Latina. El Perú, tuvo como referentes a México, Brasil, Chile y Honduras (Jones, 2007). El programa social Juntos, fue lanzado en el 2005, con el objetivo de promover el desarrollo del capital humano y ayudar a romper la transmisión generacional e intergeneracional de la pobreza; además, de generar condiciones en los hogares y estos, a su vez, aseguren los derechos de niños y niñas a temas de salud y educación.

Según el informe de Estrada y Perea (2008), una de las conclusiones señala que, las políticas sociales aplicadas en los noventa priorizaron programas orientados al alivio de la pobreza, los cuales se caracterizaron por ser de naturaleza temporal y pretender atenuar los costos de ajuste y necesidades de los más pobres. Si bien en este periodo se incluyeron también programas destinados a la superación de la pobreza, su aplicación no se llegó a materializar, sino hasta la siguiente década.

Dado que los programas de transferencia monetaria condicionada se constituyen en una innovación dentro de la política social peruana, es necesario que se determinen los impactos.

Para Salgado (2010), el diseño del programa mexicano en el año 2006, intenta establecer un modelo de desarrollo comunitario en coordinación con los tres niveles DIF (nacional, estatal y municipal), pero los roles de trabajo se concentran en el nacional y estatal.Y, éste último, es el que posee el papel protagónico en cuanto al diseño, manejo de presupuesto y operación del programa. Lo anterior es de utilidad en la medida que los conceptos de autogestión y participación social son complejos y conllevan a dificultades en su medición, mientras que el de proyectos condensa la organización de los grupos en un producto. Sin embargo, se corre el riesgo de perder de vista la naturaleza que, en teoría, distinguía este programa de otras iniciativas utilitaristas de participación, pues su objetivo se describía hasta el año 2008 en sus políticas y lineamientos, como el impulso a la participación y capacidad de gestión comunitaria para el desarrollo local, donde los proyectos eran solo una manifestación del objetivo deseado y los grupos de desarrollo. Es importante el análisis de programas como Comunidad Diferente, porque, en teoría, está creado específicamente para fortalecer las prácticas de organización.

Por su parte, Arroyo (2010) menciona que los principales beneficios derivados del programa, y percibidos por la población, es el programa Juntos, siendo considerado, por los beneficiarios, como un soporte central para lograr un cambio en la vida y las familias; la distribución y el nuevo orden doméstico y las nuevas actividades familiares, han permitido que los beneficiarios reconozcan que su vida actual es mucho más organizada y ordenada que antes.

Asimismo, Arroyo identifica ciertos desafíos a superar, vinculados a factores relacionados a la selección de beneficiarios, como problemas respecto a los instrumentos para medir la situación de cada familia, que fueron ajustándose progresivamente; problemas de comunicación y traslado de información sobre los principios del Programa hacia las comunidades; el desplazamiento de familias fuera de sus localidades, que no les permitió estar durante el proceso de empadronamiento; los posibles beneficiarios que no se inscribieron inicialmente debido a que dudaban de la veracidad del Programa. Finalmente, considera ciertas limitaciones para alcanzar los objetivos del programa, como la necesidad de una mayor coordinación con las autoridades y programas del propio Estado. Pues, el programa Juntos no podrá generar mayor impacto si es que no realiza un trabajo coordinado con las autoridades de la localidad, servidores de salud y educación, así como, con las diversas organizaciones presentes en la zona.

En concordancia con ello, el 7 de abril del 2005 se creó el Programa Nacional de Apoyo Directo a los Más Pobres - Juntos, como Programa de transferencias monetarias condicionadas, con la finalidad de romper con la transmisión intergeneracional de la pobreza y potenciar el capital humano de los hogares más pobres del Perú. En ese sentido, el programa entrega un incentivo económico a los hogares usuarios, condicionado al cumplimiento de compromisos adquiridos, con el programa, en materia de educación y control de la salud de los niños y niñas. 
El 20 de octubre del 2011, se creó el Ministerio de Desarrollo e Inclusión Social - MlDlS, como ente rector de las políticas nacionales en materia de desarrollo social, superación de la pobreza y promoción de la inclusión y equidad social. y en materia de protección social de poblaciones en situación de riesgo, vulnerabilidad y abandono. En virtud de las competencias atribuidas a este nuevo organismo público, se dispuso la adscripción de Juntos al Midis, la cual quedó formalizada el 1 de enero del 2012

En el marco normativo, antes expuesto, el Midis ejerce la rectoría de la política social, y tiene como mandato diseñar, coordinar y conducir las políticas y estrategias destinadas a reducir la pobreza de la población vulnerable; de este modo, orienta el conjunto de acciones del Estado peruano en materia de desarrollo e inclusión social, encaminadas a reducir la pobreza, las desigualdades, las vulnerabilidades y los riesgos sociales en aquella brechas que no pueden ser cerradas por la política social universal.

Desde el 2012, el Midis inició la implementación del modelo de desarrollo e inclusión social en sus dos campos: el alivio de la pobreza y el impulso al desarrollo a través de la generación de oportunidades, tanto en lo que se refiere a la prestación de servicios como al desarrollo de estrategias intersectoriales e intergubernamentales articuladas; asimismo, impulsa la estrategia nacional de inclusión social, "incluir para crecer", que concentra sus actividades en hogares de la población en proceso de inclusión, tomando en cuenta las necesidades de todas las fases de la vida humana.

Juntos es una unidad ejecutora adscrita al Ministerio de Desarrollo e Inclusión Social - Midis, que busca que gestantes, niñas, niños, adolescentes y jóvenes hasta los 19 años de edad, accedan informados a servicios de salud, nutrición y educación, ello se alinea con el objetivo específico general de dicho Ministerio: "contribuir con el alivio de la pobreza y potenciar el capital humano de los hogares en situación de pobreza y pobreza extrema” y contrarrestar la transmisión intergeneracional de la pobreza, conforme a la Estrategia Nacional de Desarrollo e Inclusión Social "Incluir para crecer", como programa social de los ejes 1, 2 y 3 de la ENDIS, contribuyendo a la generación de mayores oportunidades económicas sostenibles de los hogares rurales en proceso de inclusión.

Al finalizar el segundo semestre del año 2017, el programa social Juntos contaba con 763367 hogares afiliados, que comprenden a 1640604 miembros objetivo, y recibieron abono 693504 hogares, habiendo ejecutado transferencias por un total de S/. 837858096 soles.

Las teorías que ayudaron a concretar la presente investigación son: La hermenéutica, la teoría descriptiva y la teoría de la planificación; las cuales, enmarcaron el estudio para llegar a conocer la percepción que tienen los pobladores no beneficiarios del programa social Juntos.
La primera, por ser una disciplina que se ocupa del análisis y la interpretación de realidades contextuales. Como señala Geertz (1973), la explicación interpretativa centra su atención en el significado que las instituciones, acciones, imágenes, etc. Hecho que permitió recoger las percepciones que tienen las unidades de análisis consideradas por el estudio, así como la interpretación de las realidades para proponer acciones o alternativas, que permitan mejorar al programa.

La teoría descriptiva, se centra en describir los diversos fenómenos que ocurren dentro de la realidad, con lo que se identificó los principales elementos que influyen en las percepciones de los pobladores no beneficiarios del programa social Juntos, quienes con sus opiniones visualizaron realidades concretas de lo que viene sucidiendo dentro del programa, a partir del cual, se ensayó algunas recomendaciones a las instancias ligadas al tema.

Y, la teoría de la planificación, propuesta por Ahumada (1968) y Friedman (1991), se toma como complemento de las dos anteriores a partir del cual se visualizó el futuro desde una perspectiva preventiva y prospectiva, que implica estar preparados para anticiparse y afrontar los futuros escenarios contextuales.

El índice de desarrollo humano (IDH), mide el progreso medio conseguido por un país, en tres dimensiones básicas del desarrollo humano:

- Disfrutar de una vida larga y saludable, medida a través de la esperanza de vida al nacer.

- Disponer de educación, medida a través de la tasa de alfabetización de adultos y la tasa bruta combinada de matriculación en primaria, secundaria y terciaria.

- Disfrutar de un nivel de vida digno, medido a través del PIB per cápita en términos de la paridad del poder adquisitivo (PPA) en dólares estadounidenses (USA).

\section{Métodos y materiales}

La investigación realizada, tiene un enfoque mixto de tipo aplicada y nivel descriptivo, porque se describió las variables de estudio, como método general se aplicó el método científico y como método específico el método descriptivo. La población considerada para el presente estudio, es la totalidad de los pobladores mayores de 15 años con que cuenta cada uno de los ocho distritos de las cuatro provincias del valle del Mantaro, que según la clasificación de Ramírez y Fernández (2013), corresponden a los grupos poblacionales jóvenes de 16 a 24 años, adultos de 25 a 59 años y adultos mayores de 60 años a más, quienes habitan y participan activamente en las diversas actividades familiares y comunales de cada distrito, se dejó de lado a los infantes de 0 a 15 años.

La muestra seleccionada, se basa en el muestreo probabilístico del principio de equiprobabilidad, donde 
todos los pobladores mayores de 15 años tienen la probabilidad de ser seleccionados; la muestra para la investigación, estuvo compuesta por un total de 382 pobladores, varones y mujeres no beneficiarios de los ocho distritos, dos de cada uno en las cuatro provincias del valle del Mantaro, los que voluntariamente participaron en el estudio durante la visita realizada a sus domicilios.

Para la concreción de la investigación, se ha elaborado una encuesta conteniendo 33 preguntas, las tres primeras son del nivel sociodemográfico y 30 del estilo Likert, las cuales fueron aplicadas a la muestra seleccionada.

El proceso de desarrollo de la presente investigación, permitió utilizar diversas técnicas y estratégias de la metodología cualitativa y cuantitativa, que es usado hoy en día por las ciencias sociales y, se desarrolló en función a las siguientes fases:

\section{Recolección de datos}

El equipo de investigadores, conformado por cuatro personas, ha realizado visitas programadas a cada uno de los ocho distritos priorizados, donde se realizó las entrevistas con las autoridades locales y se aplicaron las encuestas a los pobladores considerados como unidades de análisis, varones y mujeres no beneficiarios del programa Juntos. El estudio ha despertado un especial interés en la gran mayoría de los pobladores no beneficiarios, quienes manifestaban sus percepciones sobre el programa con mayor amplitud, porque consideraban inadecuada la forma que se venía desarrollando, el cual, además, tuvo los siguientes niveles:

Primero. Para el nivel teórico se utilizó el método de la investigación bibliográfica, archivística y documental planteada por De La Torre y Navarro (2008), lo cual conllevó a revisar diversos documentos escritos sobre el tema de estudio, como libros, revistas, artículos y medios electrónicos, principalmente la Internet, para acceder a las páginas web y repositorios de las revistas científicas indizadas en Alicia, Scopus y Scielo, fundamentalmente, con los cuales se profundizó y amplió el marco teórico de la investigación.

Segundo. Para sacar información de campo, el instrumento utilizado fue el cuestionario, cuya fiabilidad se determinó a través de $\alpha$ de Crombach. La técnica utilizada fue la encuesta perfilada según la escala de Likert (1932), el instrumento contiene un total de 30 ítems, todas con 5 opciones: muy mala, mala, regular, buena y muy buena, las cuales fueron aplicadas a un total de 382 personas no beneficiarias del programa social Juntos; el instrumento mide opiniones y actitudes. Así mismo, se realizó entrevistas estructuradas a algunos pobladores y autoridades en cada uno de los ocho distritos de las cuatro provincias pertenecientes al valle del Mantaro, los que fueron seleccionados teniendo en cuenta la priorización del programa Juntos y la cantidad de beneficiarios que tuvieron durante estos últimos dos años. Estas actividades, en su mayoría, fueron hechas por las estudiantes, consideradas como colaboradoras en la presente investigación bajo el seguimiento y supervisión de los docentes responsables.

\section{Procesamiento de datos}

La elaboración del presente informe fue hecha de forma participativa entre los investigadores, para el cual se distribuyó las tareas y responsabilidades; primero individualmente, el cual luego se consolidó. Se utilizaron cuatro laptops para generar bases de datos con el programa Word, donde se transcribió, ordenó y analizó los resultados de las entrevistas sostenidas con los pobladores y autoridades de las áreas de estudio, donde se observaron diversas opiniones.

Los datos obtenidos con las encuestas, han sido almacenados inicialmente en el programa estadístico SPSS 24.0, generándose una base de datos y reportes del programa estadístico SPSS, a través del cual han sido procesadas, analizadas, interpretadas y reportadas en tablas y figuras; estos resultados obtenidos, fueron trasladados al programa Word que se muestran en el presente informe.

\section{Resultados}

\section{Perspectivas socioculturales de los pobladores del valle del Mantaro sobre el programa social Juntos}

Los datos que se muestran a continuación, tienen las siguientes características: las tres primeras tablas corresponden al nivel sociodemográfico y los 30 siguientes ítems a la percepción sociocultural que tienen los pobladores no beneficiarios del programa Juntos.

En la Tabla 1, se observa que casi el $40 \%$ de los pobladores del valle del Mantaro, perciben que los programas sociales implementados por el gobierno de turno son de nivel regular $(p=0,000)$, se afirma que existe diferencia significativa entre los niveles de percepción: muy mala, mala, regular, buena y muy buena, siendo la percepción predominante regular; además, se observa con un cercano al $40 \%$ de buena.

\section{Tabla 1}

Percepción de los encuestados sobre los programas sociales que implementa el gobierno

\begin{tabular}{lccc}
\cline { 2 - 4 } & Frecuencia & Porcentaje & $\begin{array}{c}\text { Porcentaje } \\
\text { acumulado }\end{array}$ \\
\hline Muy mala & 14 & 3,7 & 3,7 \\
Mal & 70 & 18,3 & 22,0 \\
Regular & 148 & 38,7 & 60,7 \\
Buena & 144 & 37,7 & 98,4 \\
Muy buena & 6 & 1,6 & 100 \\
\hline Total & 382 & 100,0 \\
\hline
\end{tabular}

En la Tabla 2, se observa que cerca del $40 \%$ de los pobladores del valle del Mantaro perciben que el apoyo que brinda el programa Juntos es bueno $(p=0,000)$, se afirma que existe diferencia significativa entre los niveles de percepción: muy mala, mala, regular, buena y muy 
buena, siendo la percepción predominante regular; además, se observa con más del $30 \%$ de regular.

Tabla 2

Percepción de los encuestados sobre apoyo que brinda el programa Juntos

\begin{tabular}{lccc}
\cline { 2 - 4 } & Frecuencia & Porcentaje & $\begin{array}{c}\text { Porcentaje } \\
\text { acumulado }\end{array}$ \\
\hline Muy mala & 21 & 5,5 & 5,5 \\
Mal & 90 & 23,6 & 29,1 \\
Regular & 124 & 32,5 & 61,5 \\
Buena & 146 & 38,2 & 99,7 \\
Muy buena & 1 & 0,3 & 100 \\
\hline Total & 382 & 100,0 & \\
\hline
\end{tabular}

En la Tabla 3, se observa que más del $40 \%$ de los pobladores del valle del Mantaro perciben que el proceso de selección de los beneficiarios para el programa social Juntos fue regular $(p=0,000)$, se afirma que existe diferencia significativa entre los niveles de percepción: muy mala, mala, regular, buena y muy buena, siendo la percepción predominante regular; además, se observa con cerca del $35 \%$ de mala.

Tabla 3

Percepción de los encuestados sobre el proceso de selección de los beneficiarios

\begin{tabular}{lccc}
\cline { 2 - 4 } & Frecuencia & Porcentaje & $\begin{array}{c}\text { Porcentaje } \\
\text { acumulado }\end{array}$ \\
\hline Muy mala & 48 & 12,6 & 12,6 \\
Mal & 129 & 33,8 & 46,3 \\
Regular & 154 & 40,3 & 86,6 \\
Buena & 50 & 13,1 & 99,7 \\
Muy buena & 1 & 0,3 & 100 \\
\hline Total & 382 & 100,0 & \\
\hline
\end{tabular}

En la Tabla 4, se observa que cerca del $50 \%$ de los pobladores del valle del Mantaro califican que la cantidad de beneficiarios del programa social Juntos fue regular $(\mathrm{p}=$ $0,000)$, se afirma que existe diferencia significativa entre los niveles de percepción: muy mala, mala, regular, buena y muy buena, siendo la percepción predominante regular; también, se observa que cerca del $30 \%$ la considera mala.

Tabla 4

Percepción de los encuestados sobre la cantidad de beneficiarios del programa Juntos

\begin{tabular}{lccc}
\cline { 2 - 4 } & Frecuencia & Porcentaje & $\begin{array}{c}\text { Porcentaje } \\
\text { acumulado }\end{array}$ \\
\hline Muy mala & 31 & 8,1 & 8,1 \\
Mal & 109 & 28,5 & 36,6 \\
Regular & 185 & 48,4 & 85,1 \\
Buena & 57 & 14,9 & 100 \\
Muy buena & 0 & 0 & \\
\hline Total & 382 & 100,0 & \\
\hline
\end{tabular}

En la Tabla 5, se observa que casi el $65 \%$ de los pobladores del valle del Mantaro califican a las personas que cuentan con recursos y son beneficiarios del programa social Juntos como una actitud muy mala $(\mathrm{p}=$ $0,000)$, se afirma que existe diferencia significativa entre los niveles de percepción: muy mala, mala, regular, buena y muy buena, siendo la percepción predominante muy mala; también, se observa que cerca del $30 \%$ lo califica de mala.

\section{Tabla 5}

Percepción de los encuestados sobre beneficiarios de Juntos con solvencia económica

\begin{tabular}{lccc}
\cline { 2 - 4 } & Frecuencia & Porcentaje & $\begin{array}{c}\text { Porcentaje } \\
\text { acumulado }\end{array}$ \\
\hline Muy mala & 245 & 64,1 & 64,1 \\
Mal & 107 & 28,0 & 92,1 \\
Regular & 18 & 4,7 & 96,9 \\
Buena & 12 & 3,1 & 100,0 \\
\hline Total & 382 & 100,0 & \\
\hline
\end{tabular}

En la Tabla 6, se observa que el $45 \%$ de los pobladores del valle del Mantaro consideran que el nivel de organización de los beneficiarios del programa social Juntos como regular $(p=0,000)$, se afirma que existe diferencia significativa entre los niveles de percepción: muy mala, mala, regular, buena y muy buena, siendo la percepción predominante regular; también, se observa que casi el $30 \%$ lo califica de buena.

\section{Tabla 6}

Percepción de los encuestados sobre el nivel de organización de los beneficiarios del programa Juntos

Frecuencia Porcentaje $\begin{gathered}\text { Porcentaje } \\ \text { acumulado }\end{gathered}$

\begin{tabular}{lccc}
\hline Muy mala & 24 & 6,3 & 6,3 \\
Mal & 70 & 18,3 & 24,6 \\
Regular & 172 & 45,0 & 69,6 \\
Buena & 114 & 29,8 & 99,5 \\
Muy buena & 2 & 0,5 & 100,0 \\
\hline Total & 382 & 100,0 & \\
\hline
\end{tabular}

En la Tabla 7, se observa que más del $40 \%$ de los pobladores del valle del Mantaro opina que las autoridades que trabajan con el programa social Juntos como regular $(p=0,000)$, se afirma que existe diferencia significativa entre los niveles de percepción: muy mala, mala, regular, buena y muy buena, siendo la percepción predominante regular; también, se observa con cerca del $30 \%$ que lo califica de mala. 
Tabla 7

Percepción de los encuestados sobre las autoridades locales que trabajan con el programa Juntos

\begin{tabular}{lccc}
\cline { 2 - 4 } & Frecuencia & Porcentaje & $\begin{array}{c}\text { Porcentaje } \\
\text { acumulado }\end{array}$ \\
\hline Muy mala & 30 & 7,9 & 7,9 \\
Mal & 110 & 28,8 & 36,6 \\
Regular & 154 & 40,3 & 77,0 \\
Buena & 80 & 20,9 & 97,9 \\
Muy buena & 8 & 2,1 & 100,0 \\
\hline Total & 382 & 100,0 & \\
\hline
\end{tabular}

En la Tabla 8, se observa que cerca del $45 \%$ de los pobladores del valle del Mantaro opinan que las actividades que desarrollan los promotores del programa social Juntos son buenas $(\mathrm{p}=0,000)$, se afirma que existe diferencia significativa entre los niveles de percepción: muy mala, mala, regular, buena y muy buena, siendo la percepción predominante buena; también, se observa que más del $35 \%$ lo califica de regular.

Tabla 8

Percepción de los encuestados sobre las actividades que desarrollan los promotores de Juntos

\begin{tabular}{lccc}
\cline { 2 - 3 } & Frecuencia & Porcentaje & $\begin{array}{c}\text { Porcentaje } \\
\text { acumulado }\end{array}$ \\
\hline Muy mala & 9 & 2,4 & 2,4 \\
Mal & 61 & 16,0 & 18,3 \\
Regular & 136 & 35,6 & 53,9 \\
Buena & 163 & 42,7 & 96,6 \\
Muy buena & 13 & 3,4 & 100 \\
\hline Total & 382 & 100,0 & \\
\hline
\end{tabular}

En la Tabla 9, se observa que más del $40 \%$ de los pobladores del valle del Mantaro considera que el pago que realiza a los beneficiarios del programa social Juntos como buena ( $\mathrm{p}$ $=0,000)$, se afirma que existe diferencia significativa entre los niveles de percepción: muy mala, mala, regular, buena y muy buena, siendo la percepción predominante buena; también, se observa que más del $30 \%$ lo califica de regular.

Tabla 9

Percepción de los encuestados sobre las actividades que desarrollan los promotores de Juntos

Frecuencia Porcentaje $\begin{aligned} & \text { Porcentaje } \\ & \text { acumulado }\end{aligned}$

\begin{tabular}{lccc}
\hline Muy mala & 20 & 5,2 & 5,2 \\
Mal & 71 & 18,6 & 23,8 \\
Regular & 122 & 31,9 & 55,8 \\
Buena & 153 & 40,1 & 95,8 \\
Muy buena & 16 & 4,2 & 100,0 \\
\hline Total & 382 & 100,0 & \\
\hline
\end{tabular}

En la Tabla 10, se observa que el 31,9\% de los pobladores encuestados considera que el nivel de organización de los beneficiarios del programa social Juntos como mala $(p=0,000)$, se afirma que existe diferencia significativa entre los niveles de percepción: muy mala, mala, regular, buena y muy buena, siendo la percepción predominante mala; también se observa contrariamente con un 31,2\% que lo califica de buena.

Tabla 10

Percepción del entorno de los beneficiarios sobre el programa Juntos

Frecuencia Porcentaje $\begin{aligned} & \text { Porcentaje } \\ & \text { acumulado }\end{aligned}$

\begin{tabular}{lccc}
\hline Muy mala & 21 & 5,5 & 5,5 \\
Mal & 122 & 31,9 & 37,4 \\
Regular & 113 & 29,6 & 67,0 \\
Buena & 119 & 31,2 & 98,2 \\
Muy buena & 7 & 1,8 & 100,0 \\
\hline Total & 382 & 100,0 & \\
\hline
\end{tabular}

En la Tabla 11, se observa que más del $40 \%$ de los pobladores del valle del Mantaro considera que el nivel de nutrición de las familias antes de ser beneficiarias del programa social Juntos era regular $(\mathrm{p}=$ $0,000)$, se afirma que existe diferencia significativa entre los niveles de percepción: muy mala, mala, regular, buena y muy buena; siendo la percepción predominante regular; también, se observa que casi el $30 \%$ lo califica de mala.

\section{Tabla 11}

Percepción de los encuestados sobre las actividades que desarrollan los promotores de Juntos

\begin{tabular}{lccc}
\cline { 2 - 4 } & Frecuencia & Porcentaje & $\begin{array}{c}\text { Porcentaje } \\
\text { acumulado }\end{array}$ \\
\hline Muy mala & 16 & 4,2 & 4,2 \\
Mal & 111 & 29,1 & 33,2 \\
Regular & 153 & 40,1 & 73,3 \\
Buena & 100 & 26,2 & 99,5 \\
Muy buena & 2 & 0,5 & 100,0 \\
\hline Total & 382 & 100,0 & \\
\hline
\end{tabular}

En la Tabla 12, se observa que casi el $35 \%$ de los pobladores del valle del Mantaro percibe hoy en día el nivel de nutrición de las familias beneficiarias del programa social Juntos es buena $(p=0,000)$, se afirma que existe diferencia significativa entre los niveles de percepción: muy mala, mala, regular, buena y muy buena, siendo la percepción predominante buena; también, se observa que cerca del $35 \%$ lo califica de regular. 
Tabla 12

Percepción de los encuestados sobre el nivel de nutrición actual de las familias beneficiarias del programa Juntos

\section{Frecuencia Porcentaje Porcentaje acumulado}

\begin{tabular}{lccc}
\hline Muy mala & 15 & 3,9 & 3,9 \\
Mal & 100 & 26,2 & 30,1 \\
Regular & 129 & 33,8 & 63,9 \\
Buena & 131 & 34,3 & 98,2 \\
Muy buena & 7 & 1,8 & 100,0 \\
\hline Total & 382 & 100,0 & \\
\hline
\end{tabular}

En la Tabla 13, se observa que cerca del $50 \%$ de los pobladores del valle del Mantaro considera que la actividad productiva de los pobladores antes de ser beneficiarios del programa social Juntos fue buena $(p=0,000)$, se afirma que existe diferencia significativa entre los niveles de percepción: muy mala, mala, regular, buena y muy buena, siendo la percepción predominante buena; también, se observa que cerca del $30 \%$ lo califica de regular.

Tabla 13

Percepción de los encuestados sobre las actividades productivas de los beneficiarios de Juntos

Frecuencia Porcentaje Porcentaje acumulado

\begin{tabular}{lccc}
\hline Muy mala & 11 & 2,9 & 2,9 \\
Mal & 75 & 19,6 & 22,5 \\
Regular & 107 & 28,0 & 50,5 \\
Buena & 186 & 48,7 & 99,2 \\
Muy buena & 3 & 0,8 & 100,0 \\
\hline Total & 382 & 100,0 & \\
\hline
\end{tabular}

En la Tabla 14, se observa que más del $35 \%$ de los pobladores del valle del Mantaro considera que hoy en día la actividad productiva de los pobladores beneficiarios del programa social Juntos es mala ( $p=0,000)$, se afirma que existe diferencia significativa entre los niveles de percepción: muy mala, mala, regular, buena y muy buena, siendo la percepción predominante mala; también, se observa que casi del $30 \%$ lo califica de regular.

Tabla 14

Percepción de los encuestados sobre las actividades productivas que desarrollan los beneficiarios del programa Juntos

Frecuencia Porcentaje $\begin{gathered}\text { Porcentaje } \\ \text { acumulado }\end{gathered}$

\begin{tabular}{lccc}
\hline Muy mala & 22 & 5,8 & 5,8 \\
Mal & 140 & 36,6 & 42,4 \\
Regular & 114 & 29,8 & 72,3 \\
Buena & 99 & 25,9 & 98,2 \\
Muy buena & 7 & 1,8 & 100,0 \\
\hline Total & 382 & 100,0 & \\
\hline
\end{tabular}

En la Tabla 15, se observa que cerca del $40 \%$ de los pobladores del valle del Mantaro considera que la preparación de alimentos de los pobladores antes de ser beneficiarios del programa social Juntos fue regular $(\mathrm{p}=0,000)$, se afirma que existe diferencia significativa entre los niveles de percepción: muy mala, mala, regular, buena y muy buena, siendo la percepción predominante regular; también, se observa que cerca del $35 \%$ lo considera buena.

\section{Tabla 15}

Percepción de los encuestados sobre la preparación de los alimentos antes de la llegada del programa Juntos

\begin{tabular}{lccc}
\cline { 2 - 4 } & Frecuencia & Porcentaje & $\begin{array}{c}\text { Porcentaje } \\
\text { acumulado }\end{array}$ \\
\hline Muy mala & 15 & 3.9 & 3.9 \\
Mal & 90 & 23.6 & 27.5 \\
Regular & 145 & 38.0 & 65.4 \\
Buena & 129 & 33.8 & 99.2 \\
Muy buena & 3 & 0.8 & 100.0 \\
\hline Total & 382 & 100,0 & \\
\hline
\end{tabular}

En la Tabla 16, se observa que más del $40 \%$ de los pobladores del valle del Mantaro considera que hoy en día la preparación de alimentos de los pobladores beneficiarios del programa social Juntos es buena ( $p$ $=0,000)$, se afirma que existe diferencia significativa entre los niveles de percepción: muy mala, mala, regular, buena y muy buena, siendo la percepción predominante buena; también, se observa que más del $25 \%$ lo considera mala.

\section{Tabla 16}

Percepción de los encuestados sobre la preparación de alimentos de los beneficiarios de Juntos en la actualidad

\begin{tabular}{lccc}
\cline { 2 - 4 } & Frecuencia & Porcentaje & $\begin{array}{c}\text { Porcentaje } \\
\text { acumulado }\end{array}$ \\
\hline Muy mala & 14 & 3,7 & 3,7 \\
Mal & 102 & 26,7 & 30,4 \\
Regular & 91 & 23,8 & 54,2 \\
Buena & 153 & 40,1 & 94,2 \\
Muy buena & 22 & 5,8 & 100,0 \\
\hline Total & 382 & 100,0 & \\
\hline
\end{tabular}

En la Tabla 17, se observa que más del $45 \%$ de los pobladores del valle del Mantaro considera que la higiene de los pobladores antes de ser beneficiarios del programa social Juntos fue regular $(\mathrm{p}=0,000)$, se afirma que existe diferencia significativa entre los niveles de percepción: muy mala, mala, regular, buena y muy buena, siendo la percepción predominante regular; también, se observa que cerca del $30 \%$ lo considera mala. 
Tabla 17

Percepción de los encuestados sobre la higiene de los beneficiarios de Juntos antes de su llegada

\begin{tabular}{lccc}
\cline { 2 - 4 } & Frecuencia & Porcentaje & $\begin{array}{c}\text { Porcentaje } \\
\text { acumulado }\end{array}$ \\
\hline Muy mala & 16 & 4,2 & 4,2 \\
Mal & 105 & 27,5 & 31,7 \\
Regular & 176 & 46,1 & 77,7 \\
Buena & 79 & 20,7 & 98,4 \\
Muy buena & 6 & 1,6 & 100,0 \\
\hline Total & 382 & 100,0 & \\
\hline
\end{tabular}

En la Tabla 18, se observa que más del $45 \%$ de los pobladores del valle del Mantaro considera que la higiene de los pobladores beneficiarios del programa social Juntos es regular $(p=0,000)$, se afirma que existe diferencia significativa entre los niveles de percepción: muy mala, mala, regular, buena y muy buena, siendo la percepción predominante regular; también, se observa que casi el $35 \%$ lo considera buena.

Tabla 18

Percepción de los encuestados sobre la higiene de los beneficiarios de Juntos en la actualidad

\begin{tabular}{lccc}
\cline { 2 - 4 } & Frecuencia & Porcentaje & $\begin{array}{c}\text { Porcentaje } \\
\text { acumulado }\end{array}$ \\
\hline Muy mala & 7 & 1,8 & 1,8 \\
Mal & 43 & 11,3 & 13,1 \\
Regular & 181 & 47,4 & 60,5 \\
Buena & 131 & 34,3 & 94,8 \\
Muy buena & 20 & 5,2 & 100,0 \\
\hline Total & 382 & 100,0 & \\
\hline
\end{tabular}

En la Tabla 19, se observa que más del $35 \%$ de los pobladores del valle del Mantaro considera que la salud de los pobladores antes de ser beneficiarios del programa social Juntos fue regular $(p=0,000)$, se afirma que existe diferencia significativa entre los niveles de percepción: muy mala, mala, regular, buena y muy buena, siendo la percepción predominante regular; también, se observa que más del $30 \%$ lo considera mala.

Tabla 19

Percepción de los encuestados sobre el nivel de salud de los beneficiarios de Juntos antes de su llegada

Frecuencia Porcentaje $\begin{aligned} & \text { Porcentaje } \\ & \text { acumulado }\end{aligned}$

\begin{tabular}{lccc}
\hline Muy mala & 18 & 4,7 & 4,7 \\
Mal & 123 & 32,2 & 36,9 \\
Regular & 143 & 37,4 & 74,3 \\
Buena & 95 & 24,9 & 99,2 \\
Muy buena & 3 & 0,8 & 100,0 \\
\hline Total & 382 & 100,0 & \\
\hline
\end{tabular}

En la Tabla 20, se observa que más del $45 \%$ de los pobladores del valle del Mantaro considera que la salud de los beneficiarios del programa social Juntos es buena $(p=0,000)$, se afirma que existe diferencia significativa entre los niveles de percepción: muy mala, mala, regular, buena y muy buena, siendo la percepción predominante buena; también, se observa que casi el $30 \%$ lo considera regular.

\section{Tabla 20}

Percepción de los encuestados sobre el nivel de salud de los beneficiarios de Juntos, en la actualidad

\begin{tabular}{lccc}
\cline { 2 - 4 } & Frecuencia & Porcentaje & $\begin{array}{c}\text { Porcentaje } \\
\text { acumulado }\end{array}$ \\
\hline Muy mala & 11 & 2,9 & 2,9 \\
Mal & 52 & 13,6 & 16,5 \\
Regular & 113 & 29,6 & 46,1 \\
Buena & 178 & 46,6 & 92,7 \\
Muy buena & 28 & 7,3 & 100,0 \\
\hline Total & 382 & 100,0 & \\
\hline
\end{tabular}

En la Tabla 21, se observa que más del $35 \%$ de los pobladores del valle del Mantaro considera que el nivel de la educación de los pobladores antes de ser beneficiarios del programa social Juntos fue buena ( $p$ $=0,000)$, se afirma que existe diferencia significativa entre los niveles de percepción: muy mala, mala, regular, buena y muy buena, siendo la percepción predominante buena; también, se observa que más del $35 \%$ lo considera regular.

\section{Tabla 21}

Percepción de los encuestados sobre el nivel de salud de los beneficiarios de Juntos, en la actualidad

\begin{tabular}{lccc}
\cline { 2 - 4 } & Frecuencia & Porcentaje & $\begin{array}{c}\text { Porcentaje } \\
\text { acumulado }\end{array}$ \\
\hline Muy mala & 15 & 3,9 & 3,9 \\
Mal & 88 & 23,0 & 27,0 \\
Regular & 135 & 35,3 & 62,3 \\
Buena & 136 & 35,6 & 97,9 \\
Muy buena & 8 & 2,1 & 100,0 \\
\hline Total & 382 & 100,0 & \\
\hline
\end{tabular}

En la Tabla 22, se observa que casi el $45 \%$ de los pobladores del valle del Mantaro considera que hoy en día el nivel de la educación de los pobladores beneficiarios del programa social Juntos es bueno ( $\mathrm{p}=$ $0,000)$, se afirma que existe diferencia significativa entre los niveles de percepción: muy mala, mala, regular, buena y muy buena, siendo la percepción predominante buena; también, se observa que casi el $25 \%$ lo considera regular. 
Tabla 22

Percepción de los encuestados sobre el nivel de educación de los beneficiarios de Juntos en la actualidad

\begin{tabular}{lccc}
\cline { 2 - 4 } & Frecuencia & Porcentaje & $\begin{array}{c}\text { Porcentaje } \\
\text { acumulado }\end{array}$ \\
\hline Muy mala & 9 & 2,4 & 2,4 \\
Mal & 70 & 18,3 & 20,7 \\
Regular & 91 & 23,8 & 44,5 \\
Buena & 171 & 44,8 & 89,3 \\
Muy buena & 41 & 10,7 & 100,0 \\
\hline Total & 382 & 100,0 & \\
\hline
\end{tabular}

En la Tabla 23, se observa que casi el $35 \%$ de los pobladores del valle del Mantaro considera que el compromiso de los pobladores antes de ser beneficiarios del programa social Juntos con el desarrollo del pueblo fue regular ( $\mathrm{p}=$ $0,000)$, se afirma que existe diferencia significativa entre los niveles de percepción: muy mala, mala, regular, buena y muy buena, siendo la percepción predominante regular; también, se observa que cerca del $35 \%$ lo considera buena.

Tabla 23

Percepción de los encuestados sobre el compromiso de los beneficiarios con el desarrollo del pueblo antes de Juntos

\begin{tabular}{lccc}
\cline { 2 - 4 } & Frecuencia & Porcentaje & $\begin{array}{c}\text { Porcentaje } \\
\text { acumulado }\end{array}$ \\
\hline Muy mala & 17 & 4,5 & 4,5 \\
Mal & 94 & 24,5 & 29,1 \\
Regular & 132 & 34,6 & 63,6 \\
Buena & 129 & 33,8 & 97,4 \\
Muy buena & 10 & 2,6 & 100,0 \\
\hline Total & 382 & 100,0 & \\
\hline
\end{tabular}

En la Tabla 24, se observa que casi el $35 \%$ de los pobladores del valle del Mantaro considera que el compromiso de los pobladores beneficiarios del programa social Juntos con el desarrollo del pueblo es malo $(\mathrm{p}=0,000)$, se afirma que existe diferencia significativa entre los niveles de percepción muy mala, mala, regular, buena y muy buena, siendo la percepción predominante mala; también, se observa que cerca del $35 \%$ lo considera regular.

Tabla 24

Percepción de los encuestados sobre el compromiso de los beneficiarios de Juntos con el desarrollo del pueblo en la actualidad

\begin{tabular}{lccc}
\cline { 2 - 4 } & Frecuencia & Porcentaje & $\begin{array}{c}\text { Porcentaje } \\
\text { acumulado }\end{array}$ \\
\hline Muy mala & 16 & 4,2 & 4,2 \\
Mal & 133 & 34,8 & 39,0 \\
Regular & 127 & 33,2 & 72,3 \\
Buena & 99 & 25,9 & 98,2 \\
Muy buena & 7 & 1,8 & 100,0 \\
\hline Total & 382 & 100,0 & \\
\hline
\end{tabular}

En la Tabla 25, se observa que cerca del $65 \%$ de los pobladores del valle del Mantaro considera que el nivel socioeconómico de los pobladores beneficiarios del programa social Juntos con el desarrollo del pueblo es regular $(p=0,000)$, se afirma que existe diferencia significativa entre los niveles de percepción: muy mala, mala, regular, buena y muy buena, siendo la percepción predominante regular; también, se observa que casi el $20 \%$ lo considera buena.

Tabla 25

Percepción de los encuestados sobre el nivel socioeconómico de los beneficiarios de Juntos

\begin{tabular}{lccc}
\cline { 2 - 4 } & Frecuencia & Porcentaje & $\begin{array}{c}\text { Porcentaje } \\
\text { acumulado }\end{array}$ \\
\hline Muy mala & 17 & 4,5 & 4,5 \\
Mal & 47 & 12,3 & 16,8 \\
Regular & 241 & 63,1 & 79,8 \\
Buena & 76 & 19,9 & 99,7 \\
Muy buena & 1 & 0,3 & 100,0 \\
\hline Total & 382 & 100,0 & \\
\hline
\end{tabular}

En la Tabla 26, se observa que más del $40 \%$ de los pobladores del valle del Mantaro considera que el nivel de utilización de los espacios saludables por los pobladores antes de ser beneficiarios del programa social Juntos fue mala ( $\mathrm{p}=0,000)$, se afirma que existe diferencia significativa entre los niveles de percepción: muy mala, mala, regular, buena y muy buena, siendo la percepción predominante mala; también, se observa que más del 35 $\%$ lo considera regular.

Tabla 26

Percepción de los encuestados sobre espacios saludables utilizados por los beneficiarios antes de Juntos

\begin{tabular}{lccc}
\cline { 2 - 4 } & Frecuencia & Porcentaje & $\begin{array}{c}\text { Porcentaje } \\
\text { acumulado }\end{array}$ \\
\hline Muy mala & 25 & 6,5 & 6,5 \\
Mal & 161 & 42,1 & 48,7 \\
Regular & 136 & 35,6 & 84,3 \\
Buena & 58 & 15,2 & 99,5 \\
Muy buena & 2 & 0,5 & 100,0 \\
\hline Total & 382 & 100,0 & \\
\hline
\end{tabular}

En la Tabla 27, se observa que casi el $35 \%$ de los pobladores del valle del Mantaro considera que los pobladores beneficiarios del programa social Juntos hoy en día fortalecieron los espacios saludables en sus hogares por lo que consideran regular $(\mathrm{p}=0,000)$, se afirma que existe diferencia significativa entre los niveles de percepción: muy mala, mala, regular, buena y muy buena, siendo la percepción predominante regular; también, se observa que cerca del $35 \%$ lo considera mala. 
Tabla 27

Percepción de los encuestados sobre el fortalecimiento de los espacios saludables de los beneficiarios de Juntos

\section{Frecuencia Porcentaje}

Porcentaje acumulado

\begin{tabular}{lccc}
\hline Muy mala & 31 & 8,1 & 8,1 \\
Mal & 130 & 34,0 & 42,1 \\
Regular & 131 & 34,3 & 76,4 \\
Buena & 84 & 22,0 & 98,4 \\
Muy buena & 6 & 1,6 & 100,0 \\
\hline Total & 382 & 100,0 & \\
\hline
\end{tabular}

En la Tabla 28, se observa que cerca del $45 \%$ de los pobladores considera que la participación en las actividades comunales de los pobladores beneficiarios del programa social Juntos es buena $(p=0,000)$, se afirma que existe diferencia significativa entre los niveles de percepción: muy mala, mala, regular, buena y muy buena. La percepción predominante fue buena y se observa que el 29,8\% lo considera regular.

Tabla 28

Percepción de los encuestados sobre La participación en las actividades comunales de los beneficiarios de Juntos

\begin{tabular}{lccc}
\cline { 2 - 4 } & Frecuencia & Porcentaje & $\begin{array}{c}\text { Porcentaje } \\
\text { acumulado }\end{array}$ \\
\hline Muy mala & 24 & 6,3 & 6,3 \\
Mal & 68 & 17,8 & 24,1 \\
Regular & 114 & 29,8 & 53,9 \\
Buena & 167 & 43,7 & 97,6 \\
Muy buena & 9 & 2,4 & 100,0 \\
\hline Total & 382 & 100,0 & \\
\hline
\end{tabular}

En la Tabla 29 ,se observa que más del $40 \%$ de los pobladores del valle del Mantaro considera que el nivel de articulación de las actividades por parte de los promotores del programa social Juntos es buena $(p=0,000)$, se afirma que existe diferencia significativa entre los niveles de percepción: muy mala, mala, regular, buena y muy buena, siendo la percepción predominante buena; también, se observa que más del $35 \%$ lo considera regular.

Tabla 29

Percepción de los encuestados sobre la articulación de las actividades del Programa hecha por los promotores de Juntos

\begin{tabular}{lccc}
\cline { 2 - 4 } & Frecuencia & Porcentaje & $\begin{array}{c}\text { Porcentaje } \\
\text { acumulado }\end{array}$ \\
\hline Muy mala & 17 & 4,5 & 4,5 \\
Mal & 50 & 13,1 & 17,5 \\
Regular & 142 & 37,2 & 54,7 \\
Buena & 162 & 42,4 & 97,1 \\
Muy buena & 11 & 2,9 & 100,0 \\
\hline Total & 382 & 100,0 \\
\hline
\end{tabular}

En la Tabla 30, se observa que más del $50 \%$ de los pobladores del valle del Mantaro considera que el nivel de cumplimiento de los compromisos adquiridos por los pobladores beneficiarios del programa social Juntos es bueno ( $\mathrm{p}=0,000)$, como motivo del accionar del capital social de los beneficiarios. Se afirma que existe diferencia significativa entre los niveles de percepción: muy mala, mala, regular, buena y muy buena, siendo la percepción predominante buena; también, se observa que más del $30 \%$ lo considera regular.

\section{Tabla 30}

Percepción de los encuestados sobre los beneficiarios del programa Juntos y el cumplimiento de los compromisos adquiridos

\begin{tabular}{lccc}
\cline { 2 - 4 } & Frecuencia & Porcentaje & $\begin{array}{c}\text { Porcentaje } \\
\text { acumulado }\end{array}$ \\
\hline Muy mala & 13 & 3,4 & 3,4 \\
Mal & 34 & 8,9 & 12,3 \\
Regular & 119 & 31,2 & 43,5 \\
Buena & 192 & 50,3 & 93,7 \\
Muy buena & 24 & 6,3 & 100,0 \\
\hline Total & 382 & 100,0 & \\
\hline
\end{tabular}

Como se señala, líneas arriba, los beneficiarios cumplen cotidianamente con los compromisos asumidos de forma obligatoria, porque el incumplimiento de ello hace que pierdan el beneficio, por lo que cumplen a cabalidad dichos compromisos.

\section{Discusión}

Sobre la percepción social que tienen los pobladores no beneficiarios sobre el programa social Juntos es positiva, teniendo en cuenta que la mayoría de los encuestados señalan que los diversos programas sociales implementados por los gobiernos de turno son de nivel regular y buena, mientras que el programa social Juntos es considerado bueno, seguido por un nivel regular. Estos datos son corroborados por las entrevistas realizadas, quienes manifiestan que es una ayuda para las personas que necesitan y, con ello, puedan salir adelante en cuanto se refiere a la salud, alimentación y responsabilidad; otros, contrariamente manifiestan que estos programas son malos, porque están mal acostumbrando a las personas beneficiarias a dejar de trabajar y se están volviendo ociosos. También señalan que se están incrementado casos de alcoholismo y favoritismo entre los beneficiarios.

Por otra parte, cuando se les pregunta sobre el proceso de selección de los beneficiarios y el número de beneficiarios que existen en cada distrito, señalan que es de nivel regular, seguido por el nivel malo en ambos casos, hecho que relacionan con actos ilícitos de corrupción y favoritismo entre las autoridades y promotores del programa. Pero cuando se les pregunta sobre las personas 
que tienen y son beneficiarios del programa Juntos, casi la totalidad de los encuestados y entrevistados manifiestan que es de nivel muy mala y mala respectivamente.

A las preguntas referidas al nivel de organización, refieren que es de nivel regular y buena en su gran mayoría, mientras referido al compromiso de las autoridades con el programa perciben en un nivel regular a mala; tal vez, esto coincide con la época electoral que se vive, ya que algunos señalan que las autoridades ya no les importa nada, porque ya están de salida; otros, manifiestan que las autoridades están más preocupados en solucionar sus problemas o en como robar y no cumplen con una gestión adecuada.

A las preguntas referidas al cumplimiento de actividades por parte de los promotores y el pago que realiza el programa a los beneficiarios, cerca del $45 \%$ de los encuestados consideran de un nivel buena, seguida de más del $35 \%$ que consideran de un nivel regular; mientras, a la pregunta referida al pago que realiza Juntos, más del $40 \%$ lo consideran buena; seguido por más del $30 \%$ que lo considera de nivel regular; y, referido al nivel de organización, los encuestados tienen una opinión dividida, porque el 31,9\% de los encuestados opinan que es mala y contrariamente; el 31,2 \% señala que es buena; pero, un 29,6\% señala que es de nivel regular.

Lo referido a las percepciones culturales que tienen los pobladores no beneficiarios sobre los beneficiarios del programa social Juntos que se viene ejecutando en el valle del Mantaro, están referidas a los cambios sufridos antes de la llegada del programa social y luego de la llegada hasta la actualidad; como lo referido al nivel nutricional, según los encuestados, la nutrición de los beneficiarios antes de la llegada del programa fue de un nivel regular, mientras que en la actualidad lo consideran de nivel buena percibiéndose una mejora en este rubro.

En cuanto se refiere a las actividades productivas, cerca del $50 \%$ de los encuestados percibían que era de nivel buena, antes de la llegada del programa, en la actualidad es considerada por más del $35 \%$ de los encuestados de mala. El cual coincide con las entrevistas realizadas que reporta que la actividad productiva de los beneficiarios ha retrocedido, porque ya no siembran sus chacras, en vez de avanzar han dejado de producir, esto a razón de no ser retirado del programa.

Sobre la preparación de alimentos antes de la llegada del programa cerca del $40 \%$ de los entrevistados manifiestan que es de nivel era regular seguida por cerca de un $35 \%$ que refiere de nivel buena, porque se comía productos naturales; mientras que en la actualidad, más del $40 \%$ refiere que es buena seguida de un nivel regular por más del $25 \%$ de los encuestados, hecho que conlleva a ser reconocido de forma positiva al programa juntos que incentiva la preparación de alimentos.

Referida a la higiene, antes del programa, es considerada de nivel regular por más del $45 \%$ de encuestados seguido por cerca del 305 que lo consideran de nivel malo. En la actualidad, más del $45 \%$ de los encuestados lo consideran de nivel regular seguido por cerca del $35 \%$ que lo considera de nivel bueno, hechos que son resultados del trabajo que vienen realizando los promotores del programa.

En cuanto se refiere a la salud, más del $35 \%$ de los encuestados refiere que fue regular, seguido por más del 30 $\%$ que refiere que fue mala; en la actualidad, más del $45 \%$ refieren que tienen una salud buena seguida por casi el 30 $\%$ que es regular, esto porque es uno de los que promueve el programa Juntos referido a la prevención y atención de la salud de los beneficiarios. Las estadísticas afirman que el número de atenciones ha crecido, pero el nivel de atención aun es limitado en los centros y puestos de salud.

Similarmente, el nivel de educación de los beneficiarios ha crecido por la presencia del programa, dado que antes era considerado de buena por más del $35 \%$ de los encuestados seguido por similar número que lo consideraba regular. En la actualidad, casi el $45 \%$ consideran al nivel educativo de los beneficiarios de buena, seguido por cerca del $25 \%$ que lo considera de nivel regular, este es otro de los rubros que incentiva el programa Juntos tan igual al de salud.

Referido al compromiso de los beneficiarios con el desarrollo del pueblo, antes era considerado por casi el $35 \%$ de regular seguido por similar cantidad que considera de nivel buena; en la actualidad, esta percepción ha bajado porque casi el $35 \%$ lo consideran de nivel mala seguido por cerca del $35 \%$ que lo consideran de nivel regular; esto ocurre, según los encuestados, porque los beneficiarios ya no quieren hacer actividades a favor de la comunidad, sino que los beneficiarios priorizan sus intereses personales. Por lo que consideran que el nivel socioeconómico de los beneficiarios ha mejorado considerablemente de lo que eran antes.

En cuanto se refiere al uso de los espacios saludables, más del $40 \%$ señalan que fue mala seguido por más del $35 \%$ que refiere de nivel regular; mientras que en la actualidad, ha bajado a menos del $35 \%$ en nivel regular y mala, hecho que conlleva a señalar que el programa debe trabajar este rubro para seguir mejorando.

Casi el $45 \%$ de los encuestados perciben que los beneficiarios participan en las actividades comunales, seguidas por casi el $30 \%$ que refieren de nivel regular. Asimismo, más del $40 \%$ de los encuetados perciben que la articulación de las actividades de los promotores es de nivel bueno, seguido por más del $35 \%$ que refieren que es regular. También, más del $50 \%$ de los encuestados consideran que los beneficiarios cumplen con sus compromisos adquiridos, seguido por más del $30 \%$ que refieren que cumplen regularmente.

Como se puede observar en los resultados, existen hechos preocupantes donde hay una tendencia de retroceso y conformismo de los beneficiarios que es percibido por los encuestados. Mientras que en otras, se observa cambios progresivos de lo que eran antes. 


\section{Conclusiones}

- La percepción sociocultural que tienen los pobladores sobre el programa social Juntos en el valle del Mantaro de la región Junín, están dicotomizadas, entre los que opinan con tendencia positiva o negativa sobre los beneficiarios, siendo corroborada con las hipótesis específicas.

- La percepción social que tienen los pobladores no beneficiarios sobre el programa social Juntos es positiva, porque la mayoría de los encuestados señalan que es de nivel regular secundado por un nivel bueno cuando se refieren a los diversos programas sociales implementados, la cantidad de beneficiarios del programa social Juntos, y nivel de organización que tienen los beneficiarios. Hechos rescatables que viene promoviendo el programa social Juntos que son percibidos por los beneficiarios.

- Otro grupo de encuestados señalan de un nivel bueno, seguido por un nivel regular; cuando se les pregunta sobre el programa social Juntos y de las actividades que desarrollan los promotores y la nutrición que tienen hoy los beneficiarios. Mientras lo referido a la selección o focalización de los beneficiarios, el trabajo que viene realizando las autoridades y el nivel de nutrición que tuvieron antes es considerada de nivel regular seguido por un nivel malo. Hechos que, en vez de avanzar, tienen una mala imagen de tendencia negativa.

- Pero además, el rubro referido a los beneficiarios que cuentan con recursos es considerado muy mala y mala casi por la totalidad de los encuestados; en ambos casos, la tendencia es negativa. Corroborando de esta forma la primera hipótesis específica planteada en la investigación.

- La percepción cultural que tienen los pobladores no beneficiarios sobre el programa social Juntos, en su mayoría, es de tendencia negativa en el valle del Mantaro, porque la mayoría de los encuestados responden de nivel buena seguido por un nivel regular y un nivel malo en algunos ítems, referido a las actividades productivas que hoy desarrollan los beneficiarios, la alimentación que tienen hoy, la higiene que tuvieron antes, el cuidado de la salud de antes y hoy, el nivel de educación de antes y hoy, el uso de los espacios saludables hoy y la participación en actividades comunales antes y hoy. Hechos preocupantes donde hay una tendencia de retroceso y conformismo de los beneficiarios que es percibido por los encuestados.

- Por otra parte, hay un grupo de encuestados que señalan una tendencia positiva cuando se refieren a las actividades productivas de antes, alimentación de antes, la higiene de hoy, cumplimiento de compromisos pactados antes y hoy, el nivel socioeconómico de los beneficiarios y el uso de espacios saludables. Aspectos rescatables del programa social Juntos que vienen promoviendo la mejora y crecimiento de los beneficiarios.

\section{Referencias bibliográficas}

Ahumada, J. (1968). La planificación del desarrollo. Santiago: ICIRA.

Arroyo, J. (2010). Estudio cualitativo de los efectos del programa Juntos en los cambios de comportamiento de los hogares beneficiarios en el distrito de Chuschi: Avances y evidencias. Lima: Programa Juntos.

Bertoni, M. y. (2010). Percepciones sociales ambientales. Estudios y perspectivas en turismo, 835-849.

Jones, N. (2007). El programa Juntos y el bienestar de la infancia. En N. d. Melenio, Relaciones con condiciones: El Estado peruano frente a su infancia (págs. 53-85). Lima: Niños del Milenio.

Kast, Freemont \& James Rosenzweig (1998). Administración en las organizaciones: enfoque de sistemas y de contingencias. Ciudad de México: Mc. Graw Hill.

Ludwig Huber, Patricia Zárate, Anahí Durand, Óscar Madalengoitia y Jorge Morel (2009). Programa Juntos: Certezas y malentendidos en torno a las transferencias condicionadas, estudio de caso de de seis distritos rurales del Perú. http://www.juntos.gob.pe/images/noticias/2011/01/Programa_Juntos_Certezas1.pdf

Merleau-Ponty, M. (1985). Fenomenología de la percepción. Barcelona: Planeta Agostini.

MIDIS (2013). Estrategia Nacional Crecer para incluir. Lima: MIDIS. http://www.MIDIS.gob.pe/ estarategias/2013/juntos

Ministerio de Desarrollo e Inclusión Social (2006). Plan Operativo Institucional y Plan de Estrategia e Implementación 2006 - Juntos. http://www.juntos. gob.pe/images/noticias/2011/01/pl an_operativo_2006.pdf

Ministerio de Desarrollo e Inclusión Social (2011). Programa Nacional de Apoyo Directo a los Más Pobres - Juntos. http://www.juntos.gob.pe/? page_id=3630.

Ministerio de Desarrollo e Inclusión Social (2012). Manual de organización y funciones del Programa Nacional de Apoyo Directo a los Más Pobres, Juntos. http://www. juntos.gob.pe/images/transparencia/RM_176_ 2012MIDIS.pdf.

Ministerio de Desarrollo e Inclusión Social (2013a). Lineamientos básicos de la política de desarrollo e inclusión social. http://www.midis.gob.pe.

Ministerio de Desarrollo e Inclusión Social (2013b). Plan Operativo Institucional 2012 - Reformulado. Programa Nacional de Apoyo a los Más Pobres, Juntos. http://www.peru.gob.pe/docs/PLANES/13117/ 
PLAN_13117_Plan_OperativoInstitucional_2013_(Reform ulado)_2013.pdf

Ministerio de Desarrollo e Inclusión Social (2015). Plan estratégico del Programa Nacional de Apoyo Directo a los Más Pobres 2011 - 2015. http://www.juntos.gob.pe/ images/noticias/2011/07/ RES-38- DE-2011.pdf

Pérez y Gardey, (2008) Percepción social. https://definicion.de/percepcion-social/

Perova, Elizaveta y Renos Vakis (2009). Impacto potencial del programa Juntos en Perú, 2005-2009.http://www. juntos.gob.pe/images/noticias/2011/01/El-Impacto-Potencial-de-JUNTOS-BancoMundial.pdf

PNUD (1990) Informe del desarrollo Humano.

PUCP. (2017) La gerencia social en la práctica. Análisis de los modelos de gestión de programas y proyectos sociales. Lima.

Rojo y Fernández (2011), Introducción. Población mayor y calidad de vida desde la perspectiva individual. Instituto de Economía, Geografía y Demografía. España.

Salgado (2010). Autogestión comunitaria en programas de desarrollo social comunidad diferente en Nuevo León y Baja California 2008-2010. México.

Sánchez, Alan y Jaramillo, Miguel (2012). Impacto del programa Juntos sobre la nutrición temprana. http://www.bcrp. gob.pe/docs/Publicaciones/DocumentosDeTrabajo/2012/documento-de-trabajo-01- 2012.pdf.

Surralles, A. (2002). De la percepción en antropologia. Algunas nociones sobre la persona dese los estudios amazónicos. Indiana, 59-72. 